\title{
SYMPOSIUM ON THE DEPOSITION OF ICE ON EXPOSED SURFACES
}

\author{
(Joint Meeting of the Society and the Institute of Refrigeration \\ held at the Royal Geographical Society's house on 30 March 1950 )
}

Mr. G. Seligman (British Glaciological Society) opened the meeting and welcomed members of the Institute of Refrigeration and representatives of the Royal Aircraft Establishment, the Admiralty, the London Transport Executive and those of several industrial undertakings. He extended a special welcome to Dr. Vincent J. Schaefer of the General Electric Company, Schenectady.

Dr. Ezer Griffiths, F.R.S. then took the Chair for the reading of papers by Messrs. J. K. Hardy, D. H. Coombs, G. G. Lilley and F. S. Sutherby.

\section{THE PHYSICS OF THE DEPOSITION PROCESS}

\author{
By J. K. HARDY \\ (Royal Aircraft Establishment)
}

\section{(i) The Effect of Frost on Heat Transfer to Refrigerated Surfaces}

Heat is extracted from refrigerated chambers by a cooler through which the air in the chamber is circulated. It is transferred from the air to the cold surfaces, which are kept cold by a refrigerating machine. If temperatures approaching freezing or below are to be maintained in the chamber, the cooler, regarded as a heat exchanger, must be relatively clumsy and inefficient because of the effect of frost which forms on the cold surfaces. The frost is formed in part from humid air which enters the chamber from outside, but in the main by distillation from whatever produce is being stored in the chamber. The rate of formation is governed as much by the nature and quantity of the material being stored as by the characteristics of the cooler. The rate of frosting and the effect of frost on the performance of the cooler can be predicted by calculation, if the condition of the air as it enters the cooler, as determined by the material being stored, is known.

When air flows over a surface which is at a lower temperature, heat is transferred to the surface by convection. If conditions are such that water condenses on the surface, the rate of transfer of heat is increased by the latent heat of condensation; when frost forms, this is further increased by the latent heat of fusion. The total rate at which heat is transferred is the sum of the two components, sensible heat and latent heat, and, except for the effect which the total may have on the temperature of the surface, each may be calculated independently. Data on the transfer of sensible heat by convection are extensive; these may be used, also, to calculate the rate of the transfer of latent heat by condensation because the coefficients of transfer are related. The calculation of rate of condensation is no more difficult than that of the rate of transfer of heat by convection.

In the case of water vapour, it so happens that the diffusivity of the vapour in air is almost identical with the diffusivity of heat. This is the reason for the simplicity of the psychromatic equation as used in wet and dry bulb hygrometry. In consequence of this, the coefficient of transfer of heat, if expressed in an appropriate manner, will have the same value as the coefficient of transfer of water by condensation, or evaporation. The coefficient of transfer of heat, $k_{h}$, is defined by the equation which gives the rate of transfer per unit area, namely

$$
536
$$




$$
H=k_{h} \rho V C_{p}\left(t_{s}-t_{a}\right) *
$$

The corresponding equation which gives the rate of condensation is

$$
W=k_{u} \rho V\left(\frac{e_{s}-e_{a}}{P}\right) \times 0.622
$$

These equations will be understood more easily, perhaps, if the more familiar conductance of heat, $h$, as occurs in Nusselt's Number, is substituted for the coefficients $k_{w}$ and $k_{h}$.

We have

and, for water vapour in air,

$$
\begin{aligned}
h & =k_{h} \rho V C_{p} \\
k_{w} & =k_{h}, \\
W & =\frac{h}{C_{p}}\left(\frac{e_{s}-e_{a}}{P}\right) \times 0.622
\end{aligned}
$$

so

The derivation of these equations is discussed at greater length in reference $\mathrm{I}$.

The rate at which water is deposited, in the form of frost, is calculated in exactly the same way as when, at higher temperatures, it condenses as liquid. There are, however, two practical difficulties. First, thermal conductance $h$, and the conductance of water vapour, $k_{w} \rho V$, changes because the deposition of frost causes the geometry of the cooler to change progressively. Second, the temperature of surface of the frost exposed to the air changes as the deposit grows because the frost insulates the surface thermally. It is possible, usually, to deal with the change in geometry and to calculate thermal conductance from air to surface at any stage of the frosting-in the case of pipe banks, for instance, the data of reference 2 are sufficient. The temperature of the exposed surface of the frost can be calculated if the thermal conductivity of the frost can be determined.

From experiments with banks of pipes, K. C. Hales has found how the density of the frost is related to the conditions of operation of the cooler, and has related density and thermal conductivity. His empirical equation which gives the density of the frost is

$$
\rho_{s}=C \sqrt{ } V \cdot W^{-0.3}
$$

with $\rho_{s}$ in lb./cu. ft., $V$, the velocity of the air at the point of maximum constriction between pipes in $\mathrm{ft} . / \mathrm{sec}$., and $W$, the rate of frosting, in $\mathrm{lb} . / \mathrm{hr} . / \mathrm{sq}$. $\mathrm{ft}$. Hales gives the value of $C$, the constant, as $\mathrm{I} \cdot \mathrm{O}$. But, it appears that there is better agreement with observed values for density with $C=\mathrm{I} \cdot \mathrm{I}$ for in-line banks and $1 \cdot 3$ for staggered banks. The thermal conductivity, which was determined indirectly, is practically the same as that of packed snow of the same density, as measured by
Devaux.

It is possible with these data to calculate the performance of a cooler at different stages in the process of frosting. The easiest method, it seems, is to select different thicknesses of frost and to calculate the performance of the cooler and, also, how long it has taken for the frost to grow to the thickness chosen.

The detrimental effect of a high loading on a cooler is shown by Hale's equation; it is doubly detrimental because the cooler will frost rapidly and, in consequence of this, the thermal conductivity of the frost will be low.

There is scope for further experiments with frosted coolers, in particular on the effect of size

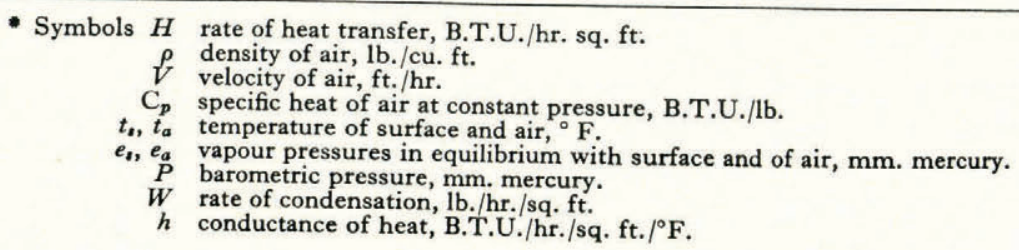


of pipe and geometrical arrangement. There is some evidence that pipes of a smaller size than conventional would be an advantage.

This paper is published by permission of the Chief Scientist, Ministry of Supply.

\section{R E F E R E N C E S}

I. Hales, K. C., Hardy, J. K., and Mann, G. Condensation of water on refrigerated surfaces. (In preparation.)

2. Grimison, E. D. Correlation and utilisation of new data on flow resistance and heat transfer for tube tanks. Trans. A.S.M.E., Vol. 59, 1937.

3. Dorsey, N. E. Properties of ordinary water substance in all its phases. Reinhold Publishing Corporation, New York, 1940.

\section{(2) ICING OF AIRCRAFT}

In certain atmospheric conditions ice forms on an aircraft whilst in flight. It is formed by droplets of water which strike all forward-facing surfaces. In severe conditions of icing the ice can grow in thickness at a rate of one to two tenths of an inch a minute. The principal effect is not the weight of the ice but the disturbance of the flow of air which causes an increase in drag and slows down the aircraft. In an extreme case, level flight cannot be maintained. The danger from icing, in all-weather operations, is such that protection against ice is a requirement for all the newer civil transports.

As has been mentioned, the ice forms from droplets of water. The water is super-cooled often to well below its nominal freezing point; icing has been reported at temperatures down to $-40^{\circ} \mathrm{F}$. $\left(-40^{\circ} \mathrm{C}\right.$.). Contrary to expectation, water in the super-cooled state, at least in the form of small drops, is remarkably stable and attempts in this country to cause freezing by ultrasonic vibrations were unsuccessful. Although it is not possible to make the droplets freeze the same effect can be produced by seeding a cloud, which is at a temperature below freezing point, with pellets of dry ice. The pellets as they fall leave in their wake very large numbers of minute crystals. These crystals grow in size at the expense of the droplets of water, because of the difference in vapour pressure between ice and water, so the cloud of droplets disperses and is replaced by a cloud of ice crystals, the latter being quite harmless so far as aircraft are concerned. The rate at which the ice crystals spread from the line of seeding is surprising. In the case of stratus cloud the rate is about two miles an hour on either side of the line of seeding.

Icing, usually, is encountered in cloud when the size of droplet is quite small, between ro and 20 microns in diameter. It can be caused by freezing rain or drizzle when the size of drop is comparatively large. In the case of small droplets in particular, the droplets are deflected by the air as it flows round the aircraft so that only a fraction of those in its path strike the surface. This fraction is called the efficiency of catch. It is greater for large drops than for small, it is greater the higher the speed and the smaller the part of the aircraft under consideration. In the case of cylinders the efficiency of catch can be calculated from the data of Langmuir and Blodgett, ${ }^{1}$ who extended earlier work on this subject by G. I. Taylor and Mrs. Glauert.

The rate at which ice will form depends on the efficiency of catch and on the concentration of water. The information on the physical constitution of conditions of icing, namely temperature, size of drops, concentration of water, has been summarized by Lewis and Jones. ${ }^{2}$ The measurements made by William Lewis were all made in the U.S.A., but there is no reason for supposing that the data will not apply generally to all other parts of the world.

The form which the ice may take depends very largely on the purely thermal effects associated with its formation. As the ice forms, the latent heat of fusion is liberated and this must be dissipated if the process is to continue. Because of the liberation of heat, the temperature of the ice is above that of the surroundings, the elevation in temperature being just sufficient to dissipate heat at a rate appropriate to the rate at which the ice is forming. The heat is dissipated both by convection and evaporation and in raising the temperature of the water from the temperature of the air to 
that of the ice. The rate at which heat is dissipated in different conditions can be calculated, and from this the rate of formation of ice may be found. Measurements in the laboratory have shown that the actual and the calculated rates agree almost exactly.

The ice which forms may be one of two broad types, rime or glaze. Rime, characteristically, has a porous structure, and, in consequence, is white in appearance and has a matt surface. It may be compact, or, when the droplets are small, it may have a loose fibrous structure composed mainly of small crystals of ice. Glaze ice is practically solid ice and, as the name implies, the surface has a glazed appearance.

With rime, the droplets of water freeze when they strike the surface with little or no spreading. This implies that the latent heat of fusion can be dissipated from the area of impact of the water. The rate of dissipation is a maximum when the temperature of the ice as it forms reaches $32^{\circ} \mathrm{F}$. $\left(0^{\circ} \mathrm{C}\right.$.), and this is the point of transition to the glaze type of ice. It is evident that much more water may be intercepted than can be frozen in the area of impact; it is the water flowing over the ice to freeze farther back which gives the ice its glazed appearance. The process is familiar in the formation of the common icicle.

The form of ice, whether glaze or rime, depends on the factors which determine the rate of dissipation of heat, namely temperature of the air, the velocity of flow and the shape and size of the part, and the factors which determine the rate of catch of water, namely concentration of water efficiency of catch and again velocity. Glaze ice is more common at temperatures near to $32^{\circ} \mathrm{F}$., the freezing point, but it occurs at temperatures down to around $0^{\circ} \mathrm{F}$. $\left(-\mathrm{r} 8^{\circ} \mathrm{C}\right.$.). Rime can form at any temperature in the icing range.

The modern method of protecting aircraft against ice is to heat the surfaces on which ice would form. On the wings, for instance, the heated area extends from the leading edge to between 5 and I 5 per cent of the' chord, the actual extent depending on the chord. This, usually, is heated by air at a temperature of around $35^{\circ} \mathrm{F}$. ( $177^{\circ} \mathrm{C}$.) which flows through shallow passages formed by a corrugated inner skin attached to the outer skin of the wing. The rate at which heat is supplied is of the order of 8,000 B.T.U./hr. per foot span $(6,609 \mathrm{~kg}$. cal. $/ \mathrm{hr} . / \mathrm{m}$.) of the wing.

An alternative, which was used to some extent by the Germans, is to allow ice to form and to shed it intermittently by heating, electrical heating mats being applied to the surface.

Windscreens and other transparencies may be kept clear of ice by heating them. This can be done electrically by using a glass which has been treated so as to make the surface conduct electricity. Or it can be done by having a double pane screen with a flow of heated air between the panels. A spray of alcohol on the exposed surface is still being used to protect windscreens against ice.

This paper is published by permission of the Chief Scientist, Ministry of Supply.

\section{R E F E R E N C E S}

r. Langmuir, I., and Blodgett, K. B. A mathematical investigation of water droplet trajectories. General Electric Co. Rep., 1945 . (Also available as U.S. Army Air Forces Tech. Rep., No. 5418.)

2. Jones, A. R., and Lewis, W. (U.S.A.) National Advisory Committee for Aeronautics. Tech. Note, No. $1855,1949$. 